\title{
Studies on a Class of AWG-Based Node Architectures for Optical Burst-Switched Networks*
}

\author{
Yurong (Grace) Huang ${ }^{1}$, Debasish Datta ${ }^{2}$, Xue Qiu ${ }^{3}$, Jing Zhang ${ }^{1}$, \\ Hyuk-Kyu Park ${ }^{3}$, Young-Chon Kim ${ }^{3}$, Jonathan P. Heritage ${ }^{1}$, \\ and Biswanath Mukherjee ${ }^{1}$ \\ ${ }^{1}$ University of California, Davis, CA 95616, yrhuang@ece.ucdavis .edu \\ ${ }^{2}$ Indian Institute of Technology, Kharagpur, West Bengal 721302, India. \\ ${ }^{3}$ School of Electronics and Information, Chonbuk National University, Jeonju, Korea. \\ yckim@chonbuk.ac.kr
}

\begin{abstract}
We investigate a class of novel node architectures based on the static arrayed-waveguide gratings (AWGs) and tunable waveband converters (TWBCs) for optical burst-switched (OBS) networks. As compared to the other AWG-based architectures using tunable wavelength converters (TWCs) for switching, our design needs much fewer wavelength-converting devices, TWBCs, instead of a large number of TWCs (operating only on one wavelength at a time). Notwithstanding the inherent simplicity, AWG-based architectures, due to the static routing properties of AWGs, exhibit some internal blocking as compared to the strictly nonblocking OBS nodes employing SOA/TWC-based architectures. We address this issue in our design methodology to arrive at different candidate node architectures using multiple layers of AWGs. Our simulation results indicate that the proposed class of node architectures using TWBCs and multiple layers of AWG can offer acceptable blocking performance with a simple and cost-effective optical hardware for OBS networks.
\end{abstract}

\section{Introduction}

A wavelength-division-multiplexing (WDM)-based network can alleviate the problem of electronic processing bottleneck by performing some of the switching/routing functionalities. Generally, there are three categories of switching technique for alloptical networks: optical circuit switching (OCS), optical packet switching (OPS) and optical burst switching (OBS) [1]-[2]. The implementation of OCS using dedicated lightpaths between source-destination pairs may result in inefficient utilization of bandwidth resources. To improve bandwidth utilization, one might employ OPS/OBS, which can provide better resource utilization by employing statistical multiplexing and traffic engineering in optical domain. In OBS, several packets are assembled into a longer packet, called burst. The header of the burst is transmitted prior to its data burst with an offset time, which enables intermediate nodes to reconfigure their switches before the burst arrives. The header is processed electronically at every node

\footnotetext{
This work has been supported in parts by NSF Grant Nos. ANI-02-07864, INT-03-23384, KOSEF, OIRC, and KIPA professorship program.
} 
and a modified header is transmitted again in optical domain for onward reservation, while the data burst propagates entirely in the optical domain from ingress to egress nodes.

As network bandwidth keeps increasing, performing routing in electronic domain through opto-electronic/electro-optic conversions become complicated and expensive as well. Hence, the implementation of transparent routing functionality in optical domain plays an important role for the evolution of optical networks. In general, for realizing routing functionalities in optical domain, OCS and OBS networks have different requirements for switch reconfiguration time. A slow switch might suffice OCS operations, since a lightpath is normally setup for a long period as compared to the switch reconfiguration time at nodes. However, for bursty traffic with finer traffic granularity, the switch reconfiguration time needs to be smaller for better network efficiency, thus requiring fast optical switching for OBS networks. In this paper, we explore some candidate switching schemes for OBS nodes, leading to a class of fast and cost-efficient node architectures for OBS routers.

Designing node architectures with fast and non-blocking switch configurations has been a major issue in OBS network implementation. Some of the recent efforts in this direction [3] have been to implement strictly non-blocking node architectures by using tunable wavelength converters (TWCs) in conjunction with semiconductor optical amplifiers (SOAs). However, the hardware complexity in such configurations has motivated other research groups to explore alternative node architectures that can offer simple and fast operation, however with a limited blocking in the switch fabric in some configurations. Most of these node architectures make use of TWCs along with arrayed-waveguide grating (AWG) as the basic switching hardware, the latter offering a static wavelength-selective routing functionality between its input and output ports [4]-[6]. In particular, AWG is a passive static device which is wavelength sensitive. When a lightwave enters at an input port of an AWG at a given wavelength, the output port wherefrom it will exit, depends on the wavelength it carries and the position of the input port. Employing this feature of AWG along with fast TWCs preceding each input port of AWG, a transparent switching unit can be realized for OBS networks. This type of switching scheme is attractive because it reduces network cost and complexity, and simplifies network management, as compared to the network architectures employing a large number of SOAs for switching purpose. Furthermore, because of its passive nature, AWG is a highly-reliable device without any power consumption and also offers lower insertion loss than a normal space switch [6].

In view of the above, we propose in this paper, a novel class of AWG-based OBS node architectures, wherein we reduce the optical hardware requirement furthermore by using the concept of waveband conversion (discussed later in details). We examine the performance of proposed node architectures in terms of burst-rejection probability and node throughput, and compare with the performance of OBS nodes with strictly non-blocking architecture. We also provide a methodology to improve the blocking performance with larger number of AWGs. 


\section{Strictly-Nonblocking OBS Node Architectures}

Generally, any switching unit in a network node can be categorized in terms of its blocking performance in presence of contentions between incoming packets/bursts/ calls (bursts in case of OBS networks). In particular, in a switching unit, contention might occur when two or more bursts want to exit the switch though the same output port at the same time. Indeed, one of the contending bursts can only win the contention, while the others are lost if the node does not have any buffer to store them during the period of contention. However, besides the natural blocking resulting from this output-port contention, a switching unit might exhibit another type of blocking, called internal blocking, when a burst loss occurs even in absence of output-port contention. Such losses occur due to the lack of alternative paths between input and out pout ports due to limitation in physical connectivity within the switch. However, the nodes that offer strictly non-blocking (i.e., no internal blocking) must employ an exhaustive connectivity between input and output ports with a complex hardware. In OBS nodes, similar issues become relevant, and the switching scheme proposed earlier in [3] using wavelength converters and SOAs offer strictly-nonblocking performance but with a high hardware complexity, and also suffer from lower reliability due to the use of large number of active devices such as tunable wavelength converters (TWCs) and SOAs. More recent switching schemes for OBS nodes [4]-[6] adopt simpler optical hardware using AWGs along with TWCs, however with limited internal blocking. In the following section, we propose a class of AWG-based node architecture, albeit with novel improvisations, which reduce internal blocking but with a significantly less optical hardware for OBS routing.

\section{OBS Node Architectures Using Arrayed Waveguide Grating (AWG)}

As mentioned earlier in Section 1, AWGs can be used in conjunction with TWCs to realize switching functionality in an OBS node, however, with a limited blocking. Such node architecture has been employed in [5] as shown in Fig. 1 (a). Although TWCs preceding the AWG help in choosing alternate paths through AWG by changing the incoming wavelengths to other suitable wavelengths, the static connectivity within AWG leads to some internal blocking. To minimize this internal blocking, one can use (as shown in Fig. 1 (b)) fixed wavelength converters (FWCs) at the output stage of the switch (i.e., following AWG) along with the TWCs placed at the input stage [7]. The cost and hardware complexity of such switching fabric becomes high due to the large number of TWCs and FWCs at the input and the output stages, respectively. This motivates us to employ sharing of the wavelength conversion process among multiple wavelengths (channels) over a given band of wavelengths, which we call as waveband conversion in our proposed switching schemes. In this section, we first discuss the basic operation of TWBC as well as typical AWG routing functionalities, followed by the node architectures utilizing agile TWBCs along with static AWGs. 


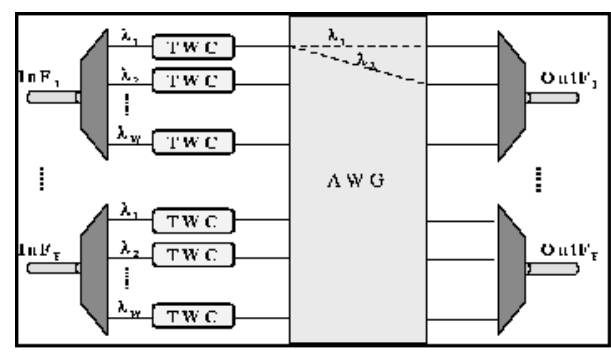

(a)

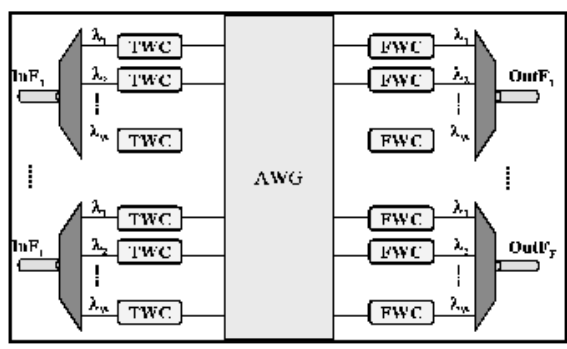

(b)

Fig. 1. (a) TWC/AWG based switching node with internal blocking; (b) TWC/AWG/FWC based nonblocking switching node

\subsection{Tunable Waveband Converter (TWBC): Basic Features}

Many efforts have been reported on the wavelength conversion techniques for one single wavelength channel [8]. Amongst them, the technique of parametric wavelength conversion offers also multi-wavelength conversion capability (i.e., concurrent conversion of a given multiple wavelengths to another set of multiple wavelengths) [8]. In this conversion scheme, the nonlinear interaction between pump lightwave $\left(f_{p}\right)$ and signal lightwave $\left(f_{s}\right)$ results in the converted wave $\left(f_{c}\right)$ at the frequency of $f_{c}=f_{p}-f_{s}$. As shown in Fig. 2, while the pump laser converts signals between $f_{1}$ and $f_{4}$, centered around $f_{p} / 2$, the conversion between $f_{2}$ and $f_{3}$ can be achieved concurrently as well. Thus, by employing a tunable pump laser, a TWBC could provide a wide range of waveband conversion. Having such conversion scheme, a given waveband could be converted to another specified waveband with a single optical device. We make use of these TWBCs along with AWGs for arriving at some viable switching schemes for OBS nodes architecture.

In the proposed switching schemes, we first divide the entire range of wavelengths (say, $W$ wavelengths) into a number ( $K$, say) of groups called wavebands, each consisting of an equal number of contiguous wavelengths $(M=W / K)$. Next, each data burst is split into $M$ segments at the ingress node, and all the burst segments are transmitted simultaneously using $M$ wavelengths from anyone of the $K$ wavebands with a duration, that is shrunk by a factor of $M$ with respect to the original burst duration with single-wavelength transmission. Once these bursts arrive at the AWG input of a node, switching functionality to route them to different possible output ports can be realized by using TWBCs for each waveband instead of TWCs for each incoming wavelength. Thus, TWBCs being comparable to TWCs in respect of hardware implementation, the number of equivalent wavelength-converting devices for each input fiber port is decreased from $W$ (number of TWCs) to $K$ (number of TWBCs) with $K=W / M$, thereby leading to a significant reduction in hardware. 


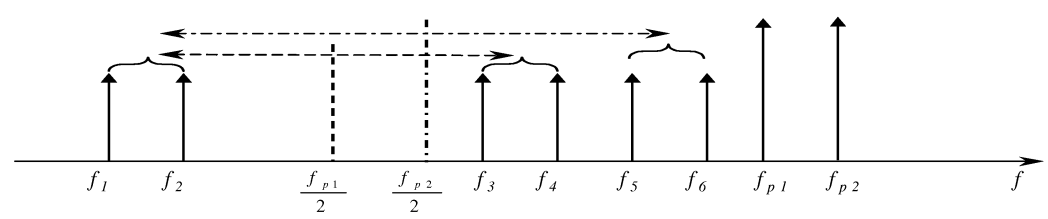

Fig. 2. A simple example for waveband conversion.

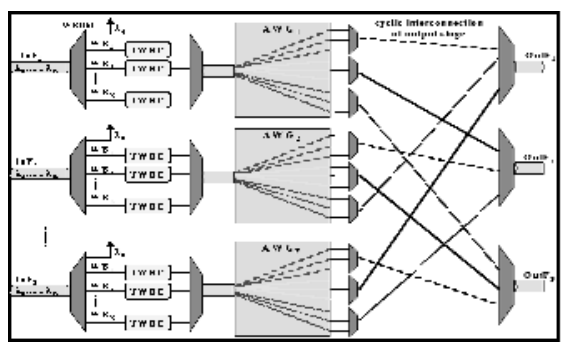

Fig. 3. A sample of WBA-SL architecture

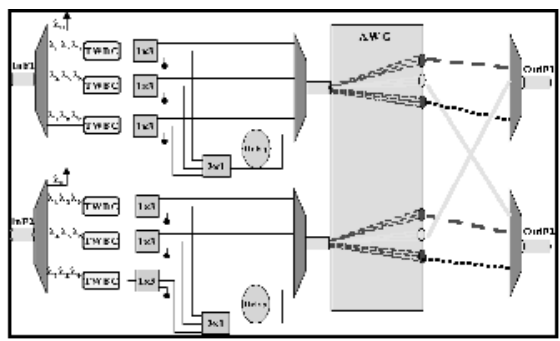

Fig. 4. A design of WBA-SL with FDL architecture

\subsection{Proposed Node Architectures Using TWBC/AWG-Based Switching Schemes}

In this subsection, we explore some novel switching schemes by combining capabilities of TWBCs and AWGs, which we call hereafter as waveband-AWG (WBA) switching. In particular, we present two new switching schemes based on WBA, namely WBA with single layer of $A W G$ (WBA-SL) and WBA with multiple layers of $A W G$ (WBA-ML).

A node is assumed to have $F$ input fiber ports and $F$ output fiber ports, with each input as well as output fiber port carrying $W$ data wavelengths (or, more precisely, $K$ $=W / M$ wavebands). First we consider the WBA-SL architecture. The proposed WBASL architecture employs, for each input fiber port, one waveband demultiplxer (WBDM), followed by $K$ TWBCs for the input stage, and one AWG at the core stage, thus requiring $F$ AWGs, $F$ WBDMs and $F K$ TWBCs in total (see Fig. 3). The output ports of $K$ TWBCs for each input fiber port are combined together again (following appropriate waveband conversion) and connected to one of the appropriate input ports of the AWG, assigned for the corresponding input fiber port. Since each incoming burst is received on one of the $K$ wavebands, they are all routed (by demultiplexing) to $K$ distinct output ports of WBDM, which are subsequently combined (or multiplexed) again and fed into the relevant AWG. A data burst arriving on a certain waveband at a given input fiber port, is converted, if necessary, to such a required waveband, that the incoming data burst can be routed by the respective AWG to the desired output fiber port. This requires, in turn, a judicious interconnection between each of the $F$ AWGs with all the output fiber ports. In other words, on the output side of AWGs, each AWG (corresponding to each input fiber port) needs to be interconnected to the $F$ output fiber ports, such that at least one waveband from each input fiber port can be routed to every fiber output port. This leads to a cyclic interconnection pattern between $F$ AWGs and $F$ output port fibers, as shown in Fig. 3. 


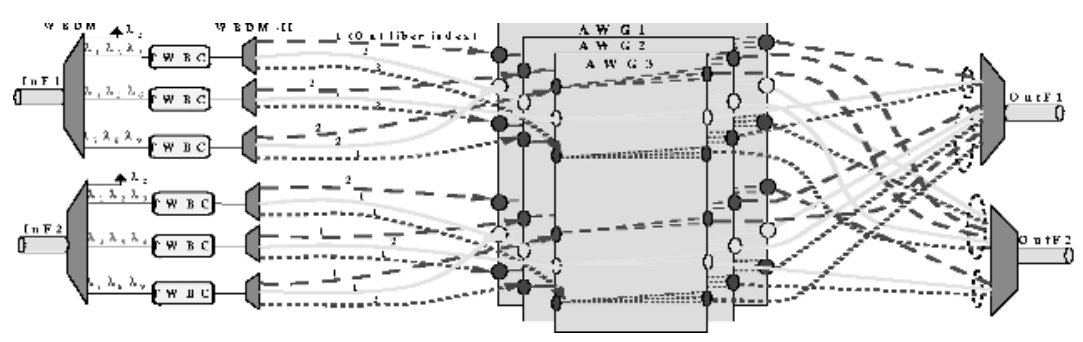

Fig. 5. The design of WBA-ML for OBS node structure

In a given input fiber port, any two or more incoming bursts destined to the same output fiber port, need to be converted to different wavebands, for being routed to the same output port without collision. However from each input fiber port, all data bursts may not be successful to reach the same output fiber port, as on the output stage, each fiber output port need to reserve at least one waveband from all the input fiber ports. In such cases, limited by the physical properties of an AWG and the output stage interconnection pattern (between AWGs and output fiber ports), burst may have to be dropped due to the internal blocking. However, such burst losses can be reduced if a delay element (e.g., FDL) can be used for each input fiber port to mitigate the contention, with the help of a $(1 \times 3)$ space switch for routing the waveband to the core stage, or to the delay element, or in the worst case to an optical termination to absorb the incoming light of the burst that lost the contention process. It may be noted that relatively shorter delay would be needed with the waveband-based transmission as compared to the single-wavelength transmission scheme. The WBA-SL architecture with FDLs for contention resolution is shown in Fig. 4 through an example of a node with 2 input/output fibers and three wavebands (i.e., $F=2, K=3$ ).

As compared to both the nonblocking OBS nodes as shown in Fig. 1 (b), and the OBS nodes employing TWC/AWG with limited internal blocking shown in Fig. 1 (a), WBA-SL architecture uses much fewer optical components, but indeed with some internal blocking. The blocking is more when one needs to avoid the $(1 \times 3)$ space switches and FDLs. However, fast space-switching of a waveband (i.e., multiple wavelengths at a time) may demand more complex optical hardware. To alleviate these limitations (use of switches and FDLs) of WBA-SL scheme, we next propose the WBA-ML architecture wherein the core stage is dilated by employing multiple layers of AWGs for each input/output fiber port (Fig. 5). In this scheme, every waveband entering an input fiber port and following TWBC can be routed to multiple AWGs, thereby utilizing the benefit of alternative paths towards output fiber ports.

In realizing the WBA-ML scheme, one can selectively route the TWBC outputs to different planes of AWGs (say, $N$ planes) using a $(1 \times N)$ space switch following each TWBC, in a manner similar to that in Fig. 4. However, space switching for waveband being difficult to realize, we propose a novel means using another stage of WBDMs following each TWBC, as shown in Fig. 5. This second or additional set of WBDMs provide a waveband-selective routing functionality for accessing different AWG planes with different wavebands, thus obviating the need of more complex $(1 \times N)$ space switching devices. For example, consider one such additional WBDM (call it WBDM-II) with one input and two output ports (following a given TWBC, assigned for a given input fiber port), corresponding to a given transmission scheme operating with two wavebands - say, red waveband and blue waveband, coming out from the 
output port 1 and the output port 2 of the WBDM-II, respectively. Also, consider that the output port 1 of WBDM-II is connected to an AWG in one plane, and the output port 2 of WBDM-II is connected to another AWG in another plane, with both AWGs being allocated for the same given input fiber port. With this setting, if the input burst to WBDM-II arrives on red waveband from the preceding TWBC, it will come out of the WBDM-II from port 1 while the input on a blue waveband will come out from port 2 of the WBDM-II. By tuning the input waveband in preceding TWBC, a burst can be routed to different output ports of the WBDM-II and hence to different planes of AWG. Thus such additional stage of WBDMs provides a switchless routing of wavebands to different planes of AWG.

It is worthwhile to note that, the connectivity between the output ports of WBDMIIs and the input ports of AWGs in different planes and the connectivity between output ports of AWGs and the fiber output ports of the node are closely related. Moreover, the connectivity on the output side would necessarily exhibit some cyclic pattern, which needs to be optimized for achieving best possible node throughput. This is one of the issues for our future study. Here, we show one possible intuitive solution for illustration, as shown in Fig. 5, wherein we have also integrated all the constituent AWGs in a given plane into one single AWG. For example, in Fig.5, each of the three AWG planes would have used two separate AWGs without the proposed integration.

As mentioned above, each plane in the proposed architecture (without AWG integration) would have $F$ AWGs, thus requiring $N \times F$ AWGs for the entire node, employing $N$ planes of AWG. However, using some inherent cyclic property of AWGs, one can integrate all the AWGs in a given plane into one composite AWG, thereby reducing the number of $\mathrm{AWG}$ devices to $F$ only, although the individual AWGs will become larger in size following the proposed. In our node design, we consider an AWG with a size of $F W \times F W$ using $F$ input/output fiber ports and supporting $W$ equally-spaced wavelengths. Such an AWG would offer a fixed (i.e., static) routing matrix, such that an optical signal on $\lambda_{i_{1}}(i=0,1, \ldots, W-1)$, at the input port $I N P_{i},(j=0,1, \ldots, F W-1)$ is routed to the output port $O P_{x}, x=(i+j) \bmod F W$. By using the input ports $j=f W, f=0,1, \ldots, F-1$, for input fiber $f$, each output port only forwards a predetermined wavelength from one input port. This allows one to reuse (spatially) the same waveband at the different input/output ports leading to the desired integration process.

\section{Illustrative Numerical Examples}

In this section, we examine our WBA-ML design to evaluate its performance in terms of node throughput and burst rejection probability due to internal blocking. The node throughput is evaluated as the ratio of the total number of bursts successfully routed to the total number of bursts arrived at the node input during a given simulation time. The node load is defined as node load $=\frac{\alpha \times L}{W \times F \times R}$, where $\alpha$ represents burst arrival rate at the node, $L$ is average burst length, $W$ is number of wavelengths per fiber, $F$ is node degree, and $R$ is per-channel transmission rate. The burst internal-rejection probability is evaluated as a ratio of the total number of rejected bursts due to an 


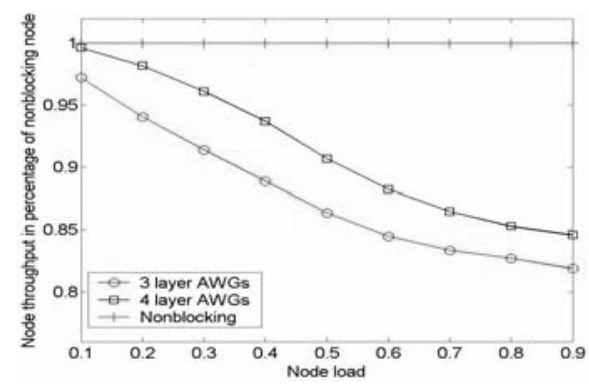

Fig. 6. Node throughput for WBA-ML architecture as percentage of the nonblocking node.

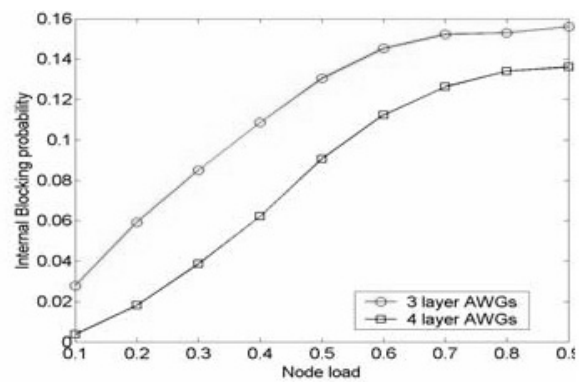

Fig. 7. Burst rejection probability due to internal blocking.

internal blocking in the switching unit to the total number of incoming bursts during a given simulation time.

For illustration purpose, we simulate a node with two input/output fiber ports $(F=$ 2) each of which carries 12 wavelengths $(W=12)$ for payload transmission. In the switching fabric, the number of AWG layers is same as the number of wavebands, $K$. A waveband in an input or output fiber where a burst arrives or departs is uniformly distributed. Both, burst arrival and burst length are assumed to be Poisson distributed.

As discussed in Section 3, the number of TWCs is significantly reduced by using TWBCs in our design for an OBS node. For example, 24 (i.e., 12 wavelengths/fiber $\times$ 2 input fiber ports) TWCs and FWCs are used in the node as shown in Fig. 1 (b), whereas in our design, 6 (i.e., 3 wavebands/fiber $\times 2$ fibers) TWBC are required. Thus, the optical hardware could be reduced significantly (in this example, by a factor of $4[1($ for $\mathrm{TWC})+1$ (for FWC) $]=8$ ). In our design, while saving cost from using TWBCs and AWGs, we might sacrifice the node performance with lower throughput and high burst rejection probability, because of the static properties of an AWG. However, as mentioned earlier, this limitation in node performance can be overcome by increasing the number of AWG layers, i.e., $K$.

Fig. 6 shows the node throughput (relative) in percentage of that of a nonblocking node (i.e., \{throughput of the proposed node / throughput of a nonblocking node $\mathrm{x}$ $100 \%$ ) as a function of the node load. We find that the proposed OBS node can achieve $89 \%$ of the throughput offered by a nonblocking node at a load of 0.4 when employing 3 layers of AWGs. The throughput can be further improved to $93.7 \%$ of a nonblocking node by using one more layer of AWG, i.e., 4 layers of AWGs. Thus, the results indicate that the node throughput can be appropriately improved by increasing the number of AWG layers. In this example, by increasing $K$ from 3 to 4 , the throughput increases by $5.4 \%$ at a load of 0.4 . It is expected that, one can approach close to the nonblocking performance with a reasonable increase in optical hardware by adding a required number of AWG layers.

The results of burst rejection probability vs. node load are plotted in Fig. 7, wherein we again observe a similar performance improvement (reduced burst rejection) with larger number of $\mathrm{AWG}$ layers. Thus by increasing the number of AWG layers, one can achieve a specified node performance, without needing significant increase in optical hardware. It may be noted that, the additional cost and complexity from a few more layers of AWGs are much less than the cost of large 
number of TWCs, replaced by much fewer TWBCs in our design. Thus, the proposed class of architectures offers a novel tradeoff towards engineering a cost-effective as well as efficient design of OBS nodes.

\section{Conclusion}

We have studied a class of novel architectures for OBS nodes, by using TWBCs along with passive AWGs with static crossconnect functionality. In comparison with other AWG-based node architectures reported so far in the literature, our node architectures offer simpler implementation with fewer optical devices, which are achieved by harnessing the novel concept of waveband conversion with TWBCs as a replacement of single-wavelength-based TWCs. Although the passive AWGs with planar integrated-optics implementation offer simpler optical hardware, their inherent static routing characteristics along with coarser granularity of TWBCs result in internal blocking in the basic WBA-SL scheme (TWBCs with single layer of AWG), which we proposed as the first and generic design step. In the next step, we improvised our basic scheme with WBA-ML scheme, wherein we employed multiple layers of AWGs along with a switchless dynamic routing scheme realized by additional stage of waveband demultiplexers. By utilizing the physical properties of AWGs, we also proposed to integrate all the AWGs in a given layer into one single AWG, without any additional degradation in blocking performance. Simulation results indicate that the proposed class of WBA-based node architectures using TWBC/AWG combination can offer acceptable blocking performance with a simple and costeffective hardware for OBS networks.

\section{References}

1. S. Verma, H. Chaskar, and R. Ravikanth, "Optical burst switching: a viable solution for terabit IP backbone," IEEE Network, Nov./Dec. 2000.

2. M. Yoo, C. Qiao, and S. Dixit, "Optical burst switching for service differentiation in the next-generation optical internet," IEEE Comm. Magazine, Feb. 2001.

3. C. M. Gauger, H. Buchta, E. Patzak, and J. Saniter, "Performance meets technology - an integrated evaluation of OBS nodes with FDL buffers," Proc. First International Workshop on Optical Burst Switching (WOBS 2003), Dallas, Texas October 2003.

4. E. Zouganeli et al., "Performance evaluation of a mesh optical burst switched network with tunable lasers and tunable wavelength converters," ConTEL Jun. 2003.

5. J. Cheyns, et al., "Performance improvement of an internally blocking optical pack/burst switch," IEEE ICC, 2003.

6. S. Bregni, A. Pattavina, and G. Vegetti, "Architectures and performance of AWG-based optical switching nodes for IP networks," IEEE JSAC, Sept. 2003.

7. S. J. B. Yoo et al., "High-performance optical-label switching packet routers and smart edge routers for the next-generation internet," IEEE JLT, Sept. 2003.

8. S. J. B. Yoo, "Wavelength conversion technologies for WDM network applications", IEEE JLT, Jun. 1996. 